\title{
Molecular characterisation and expression analysis of scavenger receptor class B member 1 (SR-B1) gene in Nile tilapia Oreochromis niloticus (Linnaeus, 1758)
}

\author{
YUCONG HUANG AND SHUANGHU CAI \\ Fisheries College, Guangdong Ocean University, Zhanjiang - 524 088, China \\ e-mail: cshcai@163.com
}

\begin{abstract}
The scavenger receptor class B member 1 (SR-B1) plays an important role in the first level of host defense against invading pathogens. In the present study, we cloned and characterised SR-B1 gene from Oreochromis niloticus (Linnaeus, 1758). The sequence of $S R$-B1 is 2248 bp long and contains a 1404 bp ORF encoded 467 amino acids. The sequence alignment showed that $S R$-B1 gene contains ten exons and nine introns. A multiple alignment analysis suggested that SR-B1 protein contains the conserved CXXS redox and GXXXG motifs and shared high similarities with that of other species. On a phylogenetic tree, SR-B1 clustered with those of other teleosts and formed a separate fish clade. Real-time quantitative PCR analysis showed that $S R$-B1 was differentially expressed in various tissues and the expression levels in spleen and intestine were significantly upregulated by Streptococcus agalactiae infection. The results suggest that $S R$-B1 may be involved in the immune response against bacteria in Nile tilapia.
\end{abstract}

Keywords: Gene expression, Oreochromis niloticus, Phylogenetic analysis, SR-B1, Streptococcus agalactiae

\section{Introduction}

The scavenger receptors (SRs) are some of the earliest discovered family members of pattern recognition receptors and could recognise and uptake polyanionic macromolecules. SRs are constitutive of some subfamily sharing the ability of modified low-density lipoprotein recognition (Meng et al., 2012). The SR subfamily can be divided into eight different classes (A-H) according to the similarity in multi-domain protein structure (Pluddemann et al., 2006). They can be expressed in different cells, such as endothelial cells, epithelial cells and myeloid cells. The SRs have been already studied in mammals, non-mammalian vertebrates, amphibians and insects (Kiefer et al., 2002).

Some studies observed that SRs could bind many microbial molecules and form first line of host defense against invading pathogens (Meng et al., 2012; Penberthy and Ravichandran, 2016). SRs are a group of receptors that play a central role in the recognition of low-density lipoprotein by oxidation or acetylation and they can participate in the removal of a broad range of foreign substances and waste materials through a variety of receptor molecules with extensive ligand specificity (Mukhopadhyay and Gordon, 2004). Studies on SRs involvement in host defense indicates that macrophage SR (MSR) binds with the purified lipid A moiety of lipopolysaccharide derived from the bacteria Escherichia coli, Vibrio cholerae and Neisseria meningitidis (Hampton et al., 1991; Pluddemann et al., 2009; Herrera et al., 2017). Furthermore, the SRs are also found to mediate the direct non-opsonic phagocytosis of several bacteria and viruses (Arredouani et al., 2006).

Nile tilapia Oreochromis niloticus (Linnaeus, 1758) is one of the important species for freshwater aquaculture in the tropical and subtropical China. Nowadays Nile tilapia has become a fish involved in intensive aquaculture and global tilapia production increased year by year to provide a globally sustainable fish food (Tanomman et al., 2013). However, bacterial diseases are one of the limiting factors for Nile tilapia culture. In particular, Streptococcus agalactiae causes mass mortalities in several species and is the etiological agents of streptococcosis (Cai et al., 2012). Unfavourable environmental conditions as well as poor management practices may stress tilapia, causing immune suppression and increased susceptibility to streptococcal infections (Ndong et al., 2007). However, only very few studies focused on molecular studies related to the immune responses in Nile tilapia.

In the present study, we report the molecular characterisation of scavenger receptor class $\mathrm{B}$ member 1 (SR-B1) in Nile tilapia. We also analysed the expression pattern of $S R$-B1 in the various tissues of Nile tilapia after challenge by $S$. agalactiae. 


\section{Materials and methods}

Experimental animals

Healthy samples of Nile tilapia weighing approximately $50 \mathrm{~g}$ were obtained from a commercial farm in Zhanjiang City of China and cultured in tanks (1000 1) with fresh running water at $28^{\circ} \mathrm{C}$ and fed to satiation daily with commercial dry pellets. Fish were anaesthetised with tricaine methanesulfonate MS222 (Sigma, Beijing, China) prior to injections and blood collection. Animal experiments were carried out in strict accordance with the regulations of local government and approved by the Ethics Committee of Guangdong Provincial Key Laboratory of Pathogenic Biology and Epidemiology for Aquatic Economic Animals (vide approval no. GDOU17-09).

Fishes (120 nos.) were injected intraperitoneally with $0.1 \mathrm{ml}$ of $S$. agalactiae having concentration of $1 \times 10^{7}$ cells $\mathrm{ml}^{-1}$ according to the method of our research group (Gan et al., 2014) and Nile tilapia group injected with $0.1 \mathrm{ml}$ of sterile phosphate buffered saline (PBS) served as control group. Injected fishes were returned to the respective tanks and fed as before. Simultaneously, three fish from each group were sampled at different time points of $0,12,24,36,48,60,72,84,96$ and $108 \mathrm{~h}$ from the control group as well as group inoculated with $S$. agalactiae.Tissue samples of head kidney, thymus, spleen, gill, liver, heart, muscle, brain, intestine and skin were collected and frozen in liquid nitrogen for RNA extraction. All analyses were performed in triplicate.

Cloning of full-length cDNA of SR-B1

Total RNA was extracted from thymus using Trizol Reagent (Invitrogen, USA) as described in the manufacturer's instructions. The cDNA synthesis was carried out based on PrimeScript Reverse Transcriptase (Takara Bio, Japan) using total RNA and used as a template for polymerase chain reaction (PCR). The partial core cDNA fragment of $S R$-B1 was amplified by performing PCR using the set of primers MidF and MidR (Table 1). The primers MidF and MidR were designed based on the conserved nucleotide sequences of $S R$ B1 from Maylandia zebra (GenBank Accession no. XP_004569457), Pundamilia nyererei (XP_005739173) and Haplochromis burtoni (XP_005929027). PCR was performed for 35 cycles at the annealing temperature of $54^{\circ} \mathrm{C}$. The amplified PCR fragment was purified using Takara Agarose Gel DNA Purification Kit and then cloned into pMD18-T (Takara Bio) and sequenced at Shanghai Sangon Biologic Engineering \& Technology and Service Co. Ltd. The sequence information (GenBank Accession no. KF638271) was used to design the primers of 3 'RACE and 5'RACE (Table 1). 3'RACE and 5'RACE were performed with 3'-Full RACE Core Set with PrimeScript ${ }^{\mathrm{TM}}$ RTase (Takara Bio) and 5'-Full RACE Kit with TAP (Takara Bio) respectively. The PCR products were then cloned and sequenced as above. Finally, sequence contigs were edited and assembled using EditSeq and SeqMan program from the DNASTAR multiple program package (Lasergene, DNA Star Inc., USA).

\section{Expression analysis of SR-B1 in healthy tissues}

The real-time quantitative PCR (qPCR) was performed to analyse the expression pattern of $S R$-B1 in different tissues of healthy Nile tilapia. Total RNA was extracted from ten tissues of healthy Nile tilapia, including head kidney, thymus, spleen, gill, liver, heart, muscle, brain, intestine and skin using Trizol Reagent and treated with DNA-free ${ }^{\mathrm{TM}}$ DNase treatment and removal kit (Ambion, Austin, TX) to digest any contaminating genomic DNA. Then the nucleic acids were quantified by NanoDrop spectrophotometer (Thermo Scientific, USA) and transcribed into first strand cDNA using Oligo dTAdpator primer (Takara). qPCR was performed with the gene-specific primers (Table 1) based on the nucleotide

Table 1 The primers used in the cloning and mRNA expression study of $S R$-B1 gene

\begin{tabular}{lll}
\hline Primers & Sequence $\left(5^{\prime} \rightarrow 3^{\prime}\right)$ & Purpose \\
\hline MidF & ATGTTGGTGTTGGGTGCATCAGT & Partial cDNA cloning \\
MidR & CGTCAGGGCTGTAGAAAGGCAAAG & \\
GSP1 & CACTGGCCTGTTCACTGTCTTTACTG & 3'RACE \\
GSP2 & TGACAGAGCTGCCGTACTGGAGG & \\
GSP3 & TCAGGGCTGTAGAAAGGCAAAGTG & 5'RACE \\
GSP4 & AGTACGGCAGCTCTGTCAGACCAT & \\
RTF & CTGGATTTATAGTGGCAGGCAGACG & qPCR \\
RTR & CAGCAGGATTGAGGACATTGAAGAAGTA & \\
$\beta$-actinF & ACACCTTCTACAATGAGCTG & Internal control \\
$\beta$-actinR & CTGCTTGCTGATCCACATCT & Genomic DNA Cloning \\
GDF & CACTACCAGTGGCTTGGCTT & \\
\hline
\end{tabular}


sequence of $S R$-B1 cDNA. Simultaneously, $\beta$-actin gene expression was measured as an internal control. PCR cycling was carried out as follows: $94^{\circ} \mathrm{C}$ for $2 \mathrm{~min}, 40$ cycles of $94^{\circ} \mathrm{C}$ for $20 \mathrm{~s}, 60^{\circ} \mathrm{C}$ for $20 \mathrm{~s}, 72^{\circ} \mathrm{C}$ for $20 \mathrm{~s}$. All experiments were performed in triplicate on 7300 Real-Time PCR System (Applied Biosystems, USA). The $S R$-B1 mRNA expression data normalised to the $\beta$-actin mRNA control, were quantified according to the methods of Livak and Schmittgen (2001).

qPCR for the expression of SR-B1 in tissues after infection with $S$. agalactiae

To determine the expression profile of $S R$-B1 in ten different tissues of Nile tilapia, total RNA extraction, cDNA synthesis and qPCR were done as described above from all the tissues at different time points from control group and group challenged with $S$. agalactiae. $\beta$-actin was used in a similar amplification reaction performed in parallel, as an internal control.

Genomic DNA isolation and cloning of the genomic $S R$-B1 gene

Genomic DNA of Nile tilapia was extracted using the Wizard Genomic Purification Kit (Promega, Madison, WI). A pair of specific primers (Table 1) was designed based on the 3'- and 5'- UTR of SR-B1 cDNA sequence. Long and accurate (LA) PCR amplification was performed with Takara LA Taq Polymerase. PCR conditions comprised $94^{\circ} \mathrm{C} 5 \mathrm{~min}$, then 30 cycles of $94^{\circ} \mathrm{C} 1 \mathrm{~min}, 58^{\circ} \mathrm{C} 1.5 \mathrm{~min}$ and $72^{\circ} \mathrm{C} 1 \mathrm{~min}$, plus a final extension at $72^{\circ} \mathrm{C}$ for $10 \mathrm{~min}$. The amplified PCR fragment was cloned into pUCm-T (Takara Bio) and then sequenced.

\section{Sequence analysis}

A sequence homology was made using the BLAST program. Protein sequence analysis was performed with the SMART software (http://smart.embl-heidelberg.de/) to predict the conserved domains. A multiple sequence alignment was computed with the ClustalX program, and a phylogenetic tree was constructed with the MEGA4 program using Neighbour Joining method (Tamura et al., 2007).

\section{Statistical analysis}

The data were analysed with one-way analysis of variance (ANOVA) by the SPSS 13.0 software, and statistical significance was defined at $\mathrm{p}<0.05$.

\section{Results}

\section{Cloning and characterisation of SR-B1}

The partial nucleotide sequence of Nile tilapia $S R$ B1 was obtained by PCR with the primers MidF and MidR. To obtain the complete coding region of the $S R$-B1
5'- and 3'-RACE were performed using the specific primers based on the partial nucleotide sequence. The nucleotide sequences of the RACE products and the original partial sequence were assembled to generate a 2248 bp full-length $S R$-B1 cDNA sequence. The sequence contains a 1404 bp ORF flanked by a 390 bp 5'-UTR and a 454 bp 3'-UTR (Fig. 1). A polyadenylation signal (AGTAAA) was found in the 3'-UTR of the sequence. The ORF is predicted to encode a protein of 467 amino acids, including two transmembrane regions located at positions 20-39 and 440-462 and eight putative N-glycosylation sites (residues 101, 107, 128, 211, 254, 309, 329, 361 and 382). A CD36 domain including residue 12 to 464 was also discovered.

The predicted amino acid sequence shared similarity with that of teleost, reptile, mammal and bird previously registered to GenBank, and contained the conserved CXXS redox and GXXXG motifs. The deduced SR-B1 amino acid sequence has the highest $95 \%$ and the lowest $53 \%$ similarities with that of Pundamilia nyererei and Sus scrofa by BLAST analysis.

Gene structure of $S R-B 1$

The genomic DNA sequence of Nile tilapia $S R$-B1 gene was isolated based on the 5'- and 3'- UTR sequences. The gene structure of $S R$-B1 was determined by aligning the genomic and cDNA sequences. The results showed Nile tilapia $S R$-B1 gene contains ten exons separated by nine introns. The exons range in size from $56 \mathrm{bp}$ to $267 \mathrm{bp}$, and the introns range from 85 to $1554 \mathrm{bp}$. A schematic gene structure of Nile tilapia $S R-\mathrm{B} 1$ is shown in Fig. 2.

Phylogenetic analysis of the Nile tilapia SR-B1 protein

A phylogenetic tree was constructed by comparing the known SR-B1 protein sequences using ClustalX and MEGA program (Fig. 3). Phylogenetic tree showed that Nile tilapia $O$. niloticus clustered on a branch separately and grouped together with the proteins of $H$. burtoni, P. nyererei, M. zebra and then some other teleosts to form a fish-specific SR-B1 clade, distinct from the other clades formed by the reptile, mammal and bird proteins.

\section{Expression of SR-B1 in healthy tissues}

Real-time PCR was carried out to analyse the expression pattern of $S R$-B1 genes in different tissues of healthy Nile tilapia (Fig. 4). The results showed that $S R$-B1 mRNA was constitutively expressed in head kidney, thymus, spleen, gill, liver, heart, muscle, brain, intestine and skin. The expression levels of SR-B1 in spleen and intestine are about five-fold and seven-fold higher respectively when compared to that in thymus, muscle and skin. The highest levels were detected in intestine and the lowest levels in heart. 


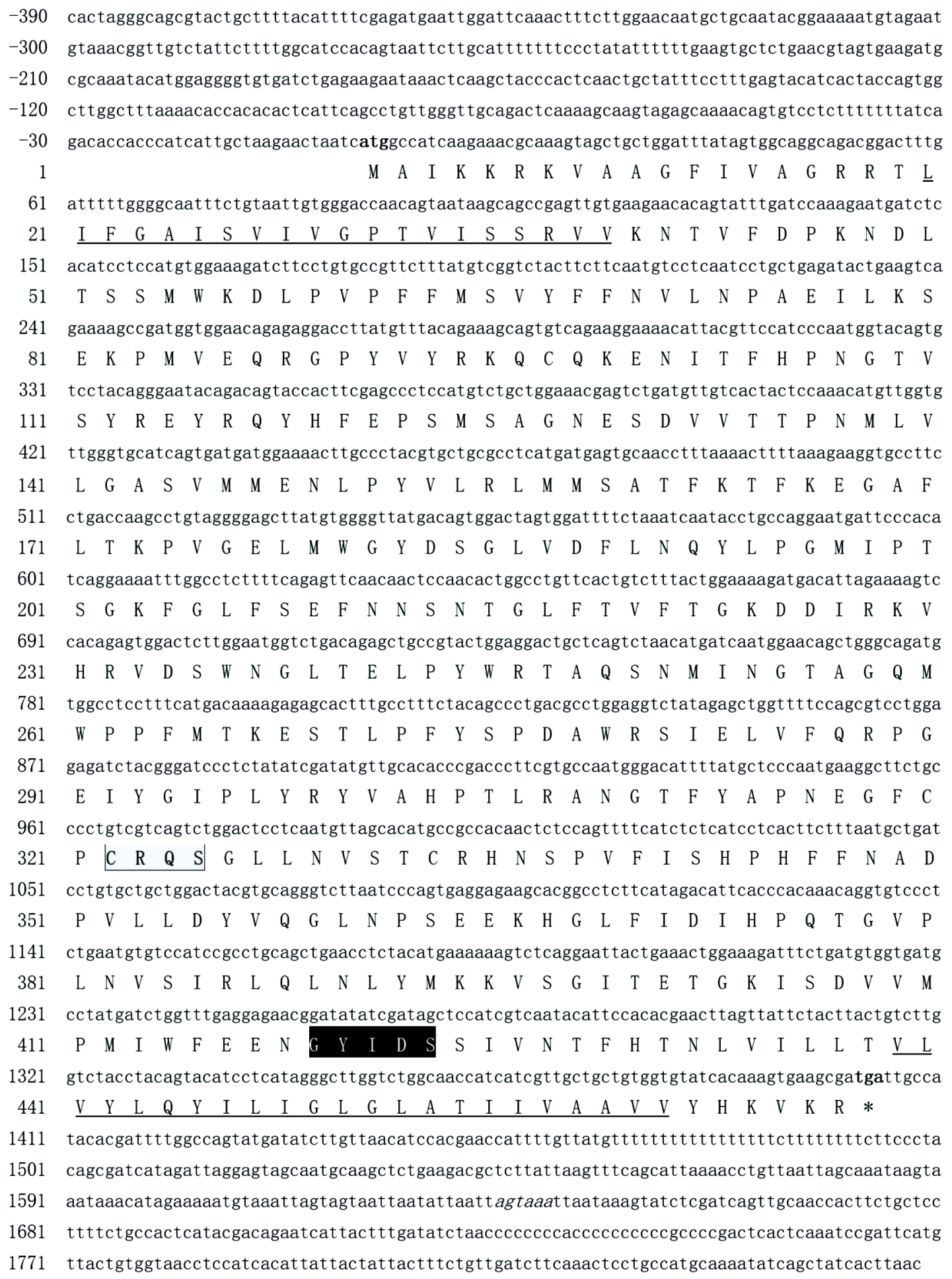

Fig. 1. Nucleotide sequence of $S R$-B1 in Nile tilapia along with the deduced amino acid sequence. The transmembrane domain is underlined. The asterisk represents the stop codon and the polyadenylation signal (AGTAAA) is denoted by italics (GenBank accession no. KF638271). The grey box in the alignment denotes a conserved CXXS redox motif and the black box denotes a conserved GXXXG motif 


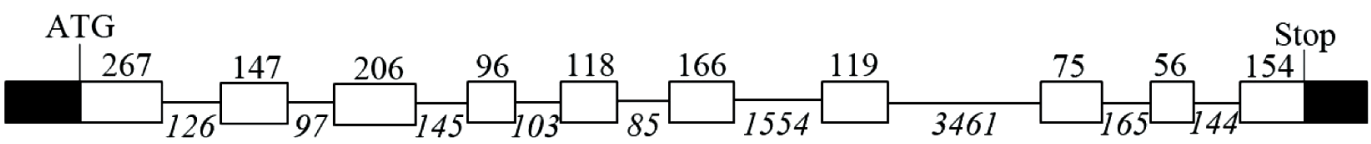

Fig. 2. The schematic gene structure of Nile tilapia $S R$-B1. Exons are indicated by white boxes, introns by black lines connecting the boxes. Numbers above the boxes and/or below the lines denote the exact length of each exon/intron in base pairs

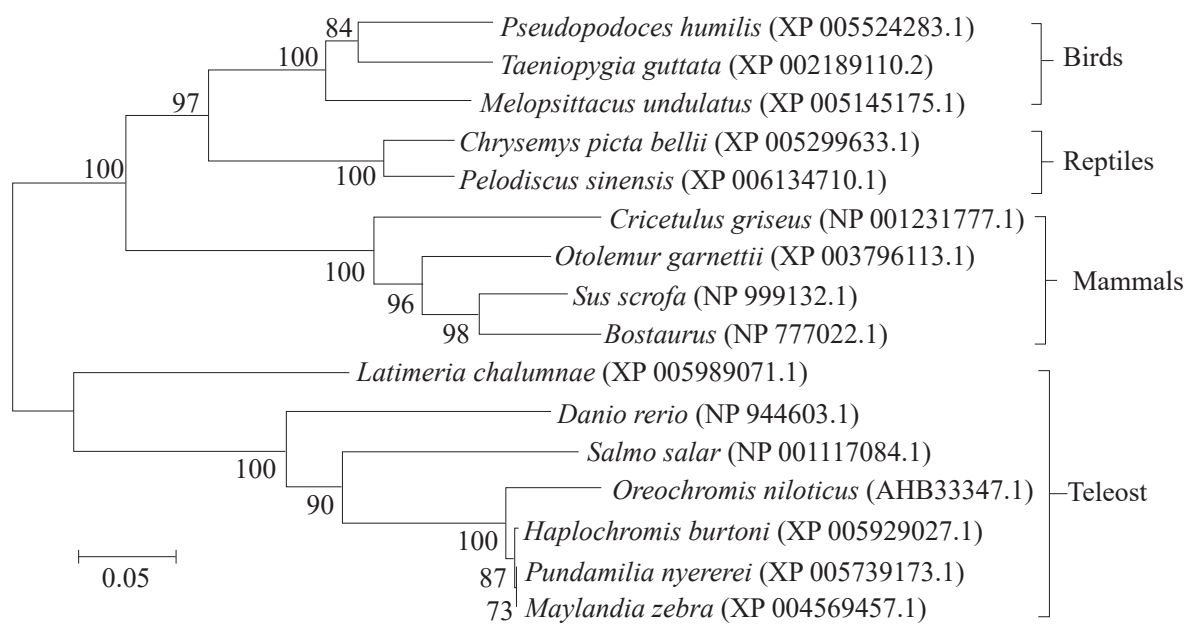

Fig. 3. Phylogenetic tree of SR-B1 protein sequences constructed with the Neighbour Joining method. The data set includes teleost, reptile, bird and mammalian sequences that are available in databases. Numbers at the nodes represent the percentage of 1000 bootstrap replications

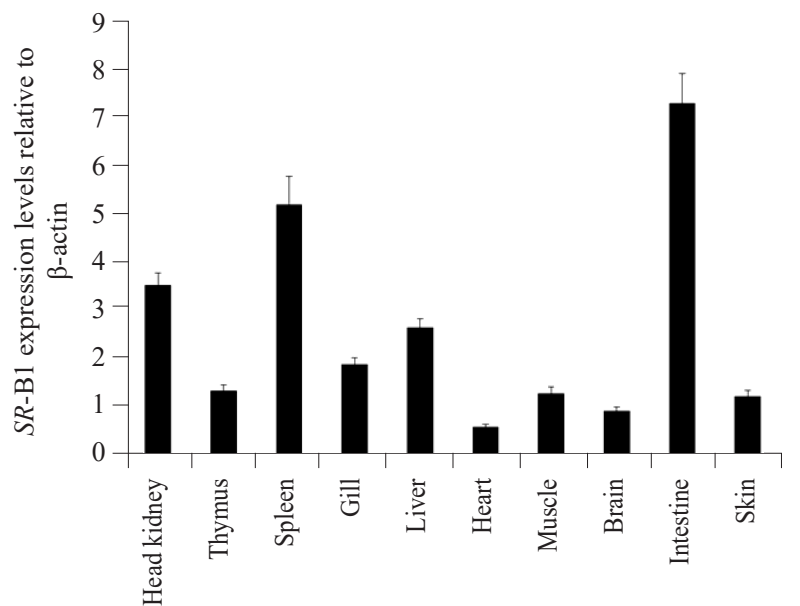

Fig. 4. Transcriptional analysis of $S R$-B1 gene in various tissues from healthy Nile tilapia. The real-time PCR was performed using primers specific for $S R$-B1 and $\beta$-actin. Expression levels of $\beta$-actin were used as internal control for real-time PCR. Each gene expression measurement was made at least in triplicate. The values are shown as means $\pm \mathrm{SE}$

Quantification of expressed SR-B1 in tissues after infection with $S$. agalactiae

qPCR was conducted to detect the mRNA levels of $S R$-B1 in various tissues at different time points of postinfection with $S$. agalactiae. The results revealed that
mRNA expression levels of $S R$-B1 gene of infection group were significantly upregulated by $S$. agalactiae induction in spleen and intestine tissues at time point of $72 \mathrm{~h}$ post-infection compared to those in head kidney, thymus, gill, liver, heart, muscle, brain and skin (Fig. 5a). The $S R$-B1 gene expression levels of infection group were up-regulated during 0-72 $\mathrm{h}$ and then dropped gradually but still slightly higher than the expression level of control group at $108 \mathrm{~h}$ in spleen and intestine (Fig. 5b, c). The $S R$-B1 expression levels of infection group in spleen are about four-fold higher and those in intestine are about six-fold, respectively when compared to those of control group when the transcript reached peak at $72 \mathrm{~h}(\mathrm{p}<0.01)$.

\section{Discussion}

The SR family consists of transmembrane proteins with multiple domains that participate in the recognition of a broad range of polyanionic ligands, including modified or oxidised low-density lipoproteins, bacteria and apoptotic cells (Kong et al., 2018). In the present study, a novel $S R$-B1 was first identified from Nile tilapia according to the homolog sequence of the teleost, adding a new member to the class A SR family. We found a highly conserved CXXS redox motif which is present in $S R$-B1 genes of all species investigated. To our knowledge, this motif has been suggested to be involved in a novel ligand-independent apoptotic pathway induced by SR-B1 through activation 


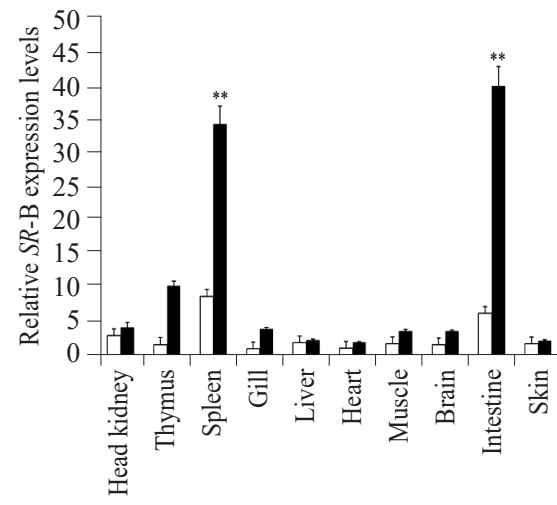

(a)

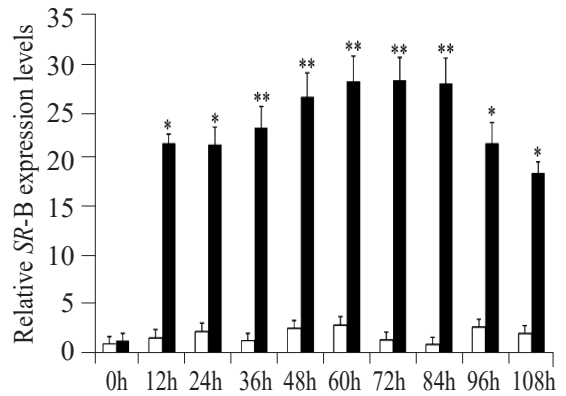

(b)

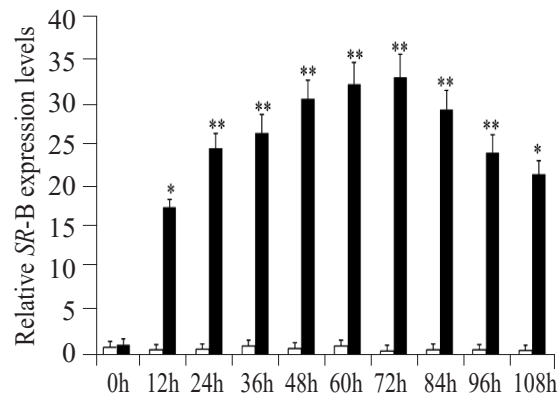

(c)

$\square$ Control group, $\square$ Infected group

Fig. 5. Expression analysis of $S R$-B1 gene in different tissues with $S$. agalactiae determined by qPCR. (a) Expression analysis of $S R$-B1 gene in various tissues at time point of $72 \mathrm{~h}$ post-infection. $S R$-B1 gene expression in (b) spleen during $108 \mathrm{~h}$ post-infection; and (c) intestine, during $108 \mathrm{~h}$ post-infection. The values are shown as means \pm S.D. Significant difference was indicated by asterisks, ${ }^{*} \mathrm{p}<0.05 ; * *, \mathrm{p}<0.01$

of the caspase- 8 pathway and regulated by endothelial nitric-oxide synthase and low-density lipoprotein ( $\mathrm{Li}$ et al., 2005). Apoptosis is an essential biological process in the development of mature multicellular organisms. Furthermore, engulfment of the dead cells is also very important in development and it raises the idea that dead cells, like microbes, are recognised by macrophages as foreign particles. SR-B1 could contribute to the clearance of apoptotic cells to prevent further inflammatory damage to neighboring cells during oxidative stress as well as during bacterial infections in Nile tilapia (Kleveland et al., 2006). There is another conserved motif GXXXG near the $\mathrm{C}$-terminal transmembrane domain. The motif is critical for the recognition, probably is associated with transport of cholesterol from peripheral tissues to the liver where it can be excreted through bile. Thus, it is found to play an important role in delivery and metabolism of high/low-density lipoprotein (Parathath et al., 2004). $S R$-B1 was found to be closely related to that of mammal, reptile and other teleosts by alignment analysis and phylogenetic tree construction. These results suggested that $S R-\mathrm{B} 1$ retained the original functional characteristics in the innate immunity of higher vertebrates during the vertebrate evolution from teleost fish to mammals.

Studies on SR-like function in mammals have also indicated an important role for this family of receptors in innate immune function. $S R-B 1(C D 36)$ is widely expressed, but the expression levels vary among different tissues, being largely restricted to lymphoid and haemapoietic lineages including monocytes, macrophages, platelets, endothelium, adipocytes, smooth muscle and certain epithelia (Zhang et al., 2013). In teleosts, SR is known to be expressed by nonspecific cytotoxic cells in the peripheral blood, spleen and anterior kidney of catfish (Qiu et al., 2013). In Nile tilapia, the expression profiles of $S R$-B1 gene differed in various tissues. The highest expression levels of $S R$-B1 gene were detected in intestine, following, spleen, head kidney and liver. Following experimental infection and challenge with the bacterial pathogen $S$. agalactiae, $S R$-B1 expression in spleen and intestine was significantly upregulated in a time-dependent manner. Currently, it is not known how closely $S R$-B1 mRNA expression reflects SR-B1 protein levels in fish. The spleen combines the innate and adaptive immune system in a uniquely organised way and intestineassociated lymphoid tissue is the major inductive site of the mucosal immune system, which is functionally independent of the systemic immune system (Cesta, 2006).

In conclusion, we have cloned and characterised $S R$ B1 gene from Nile tilapia. $S R$-B1 gene shares high structural similarity to its homologues in mammals and other teleost fish. Like other species $S R$-B1, Nile tilapia $S R$-B1 also contains an CXXS and GXXXG motif. Comparison of the mRNA expression levels in different tissues demonstrated that the $S R$-B1 gene may be involved in the host defense response. Our data confirmed that the expression levels of $S R$-B1 gene in spleen and intestine of fishes challenged with bacterial pathogen were significantly higher than in the corresponding tissues of control group fishes.

\section{Acknowledgements}

This work was supported by Natural Science Foundation of Guangdong Province (No. 2015A03030 8020 ) and Natural Science Foundation of China (No. 3130 2226). 


\section{References}

Arredouani, M. S., Yang, Z., Imrich, A., Ning, Y., Qin, G. and Kobzik, L. 2006. The macrophage scavenger receptor SR$\mathrm{AI} / \mathrm{II}$ and lung defense against pneumococci and particles. Am. J. Respir. Cell Mol. Biol., 35(4): 474-478. doi: 10.1165/ rcmb.2006-01280C.

Cai, S. H., Wang, B., Lu, Y. S., Jian, J. C. and Wu, Z. H. 2012. Development of loop-mediated isothermal amplification method for rapid detection of Streptococcus iniae, the causative agent of streptococcicosis in fish. J. Basic. Microbiol., 52(2): 116-122. doi: 10.1002/jobm.201100082.

Cesta, M. F. 2006. Normal structure, function and histology of the spleen. Toxicol. Pathol., 34(5): 455-465. DOI:10.108 0/01926230600867743.

Gan, Z., Wang, B., Lu, Y. S., Cai, S. H., Cai, J., Jian, J. C. and Wu, Z. H. 2014. Molecular characterisation and expression of CD2BP2 in Nile tilapia (Oreochromis niloticus) in response to Streptococcus agalactiae stimulus. Gene, 548(1): 126-133.

Hampton, R. Y., Golenbock, D. T., Penman, M., Krieger, M. and Raetz, C. R. 1991. Recognition and plasma clearance of endotoxin by scavenger receptors. Nature, 352(6333): 342-344. DOI: 10.1038/352342a0.

Herrera, C. M., Henderson, J. C., Crofts, A. A. and Trent, M. S. 2017. Novel coordination of lipopolysaccharide modifications in Vibrio cholerae promotes CAMP resistance. Mol. Microbiol., 106(4): 582-596. doi: 10.1111/ mmi.13835.

Kiefer, C., Sumser, E., Wernet, M. F. and Von Lintig, J. 2002. A class B scavenger receptor mediates the cellular uptake of carotenoids in Drosophila. Proc. Natl. Acad. Sci. USA, 99(16): 10581-10586. DOI: 10.1073/pnas.162182899.

Kong, T., Gong, Y., Liu, Y., Wen, X., Tran, N. T., Aweya, J. J., Zhang, Y., Ma, H., Zheng, H. and Li, S. 2018. Scavenger receptor B promotes bacteria clearance by enhancing phagocytosis and attenuates white spot syndrome virus proliferation in Scylla paramamosian. Fish Shellfish Immunol., 78: 79-90.

Kleveland, E. J., Syvertsen, B. L., Ruyter, B., Vegusdal, A., Jorgensen, S. M. and Gjoen, T. 2006. Characterisation of scavenger receptor class B, type I in Atlantic salmon (Salmo salar L.). Lipids, 41(11): 1017-1027.

Li, X. A., Guo, L., Dressman, J. L., Asmis, R. and Smart, E. J. 2005. A novel ligand-independent apoptotic pathway induced by scavenger receptor class B, type I and suppressed by endothelial nitric-oxide synthase and high density lipoprotein. J. Biol. Chem., 280(19): 19087-19096. DOI: $10.1074 / j b c . M 500944200$.

Livak, K. J. and Schmittgen, T. D. 2001. Analysis of relative gene expression data using realtime quantitative PCR and the $2^{-\Delta \Delta C}{ }_{T}$ Method. Methods, 25(4): 402-408. DOI: 10.1006/ meth.2001.1262.

Meng, Z., Zhang, X. Y., Guo, J., Xiang, L. X. and Shao, J. Z. 2012. Scavenger receptor in fish is a lipopolysaccharide recognition molecule involved in negative regulation of NF-kappaB activation by competing with TNF receptorassociated factor 2 recruitment into the TNF-alpha signaling pathway. J. Immunol., 189(8): 4024-4039. doi: 10.4049/jimmunol.

Mukhopadhyay, S. and Gordon, S. 2004. The role of scavenger receptors in pathogen recognition and innate immunity. Immunobiology, 209(1-2): 39-49. DOI: 10.1016/j.imbio.20 04.02.004

Ndong, D., Chen, Y. Y., Lin Y. H., Vaseeharan, B. and Chen, J. C. 2007. The immune response of tilapia Oreochromis mossambicus and its susceptibility to Streptococcus iniae under stress in low and high temperatures. Fish Shellfish Immunol., 22(6): 686-694. DOI: 10.1016/j.fsi.2006.08.015.

Parathath, S., Sahoo, D., Darlington, Y. F., Peng, Y., Collins, H. L., Rothblat, G. H., Williams, D. L. and Connelly, M.A. 2004. Glycine 420 near the C-terminal transmembrane domain of SR-B1 is critical for proper delivery and metabolism of high density lipoprotein cholesteryl ester. J. Biol. Chem., 279(24): 24976-24985. DOI: 10.1074/jbc. M402435200.

Penberthy, K. K. and Ravichandran, K. S. 2016. Apoptotic cell recognition receptors and scavenger receptors. Immunol. Rev., 269(1): 44-59. doi: 10.1111/imr.12376.

Pluddemann, A., Mukhopadhyay, S. and Gordon, S. 2006. The interaction of macrophage receptors with bacterial ligands. Expert Rev. Mol. Med., 8(28): 1-25. DOI: 10.1017/ S1462399406000159.

Pluddemann, A., Hoe, J. C., Makepeace, K., Moxon, E. R. and Gordon, S. 2009. The macrophage scavenger receptor A is host-protective in experimental meningococcal septicaemia. PLoS Pathog., 5(2): e1000297. doi: 10.1371/ journal.ppat.1000297.

Qiu, R., Sun, B. G., Li, J., Liu, X. and Sun, L. 2013. Identification and characterisation of a cell surface scavenger receptor cysteine-rich protein of Sciaenops ocellatus: bacterial interaction and its dependence on the conserved structural features of the SRCR domain. Fish Shellfish Immunol., 34(3): 810-818. doi: 10.1016/j.fsi.2012.12.016.

Tamura, K., Dudley, J., Nei, M. and Kumar, S. 2007. MEGA4: Molecular Evolutionary Genetics Analysis (MEGA) software version 4.0. Mol. Biol. Evol., 24(8): 1596-1599. DOI: $10.1093 / \mathrm{molbev} / \mathrm{msm} 092$.

Tanomman, S., Ketudat-Cairns, M., Jangprai, A. and Boonanuntanasarn, S. 2013. Characterisation of fatty acid 
delta-6 desaturase gene in Nile tilapia and heterogenous expression in Saccharomyces cerevisiae. Comp. Biochem. Physiol. B Biochem. Mol. Biol., 166(2): 148-156. doi: 10.1016/j.cbpb.2013.07.011.
Zhang, M., Xu, Y., Li, L., Wei, S., Zhang, S. and Liu, Z. 2013. Identification, evolution and expression of a CD36 homolog in the basal chordate amphioxus Branchiostoma japonicum. Fish Shellfish Immunol., 34(2): 546-555. 